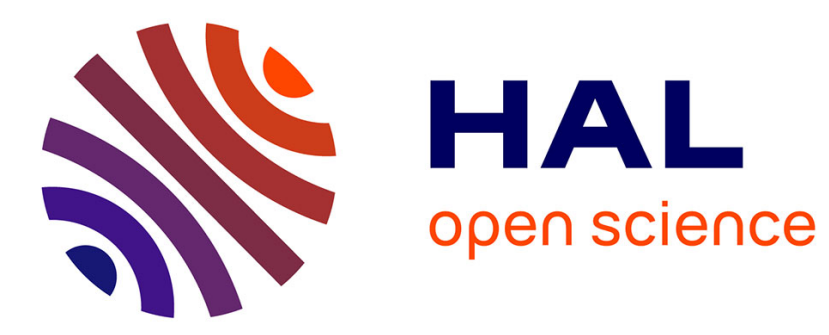

\title{
Assessing ionospheric influence on L-band SAR data: Implications on co-seismic displacement measurements of the 2008 Sichuan Earthquake.
}

Daniel Raucoules, Marcello de Michele

\section{> To cite this version:}

Daniel Raucoules, Marcello de Michele. Assessing ionospheric influence on L-band SAR data: Implications on co-seismic displacement measurements of the 2008 Sichuan Earthquake.. IEEE Geoscience and Remote Sensing Letters, 2010, 7 (2), pp.286-290. 10.1109/lgrs.2009.2033317 . hal-00509810

\section{HAL Id: hal-00509810 https: / hal-brgm.archives-ouvertes.fr/hal-00509810}

Submitted on 16 Aug 2010

HAL is a multi-disciplinary open access archive for the deposit and dissemination of scientific research documents, whether they are published or not. The documents may come from teaching and research institutions in France or abroad, or from public or private research centers.
L'archive ouverte pluridisciplinaire HAL, est destinée au dépôt et à la diffusion de documents scientifiques de niveau recherche, publiés ou non, émanant des établissements d'enseignement et de recherche français ou étrangers, des laboratoires publics ou privés. 


\title{
Assessing ionospheric influence on L-band SAR data: Implications on co-seismic displacement measurements of the 2008 Sichuan Earthquake.
}

\author{
D. Raucoules and M. de Michele
}

2 Abstract- Ionospheric contributions to the phase of L-band

3 Synthetic Aperture Radar (SAR) signals put severe

4 limitations on ground displacement measurements retrieved

5 by either differential SAR interferometry (DinSAR) or radar

6 amplitude image offsets. Such contributions result in an 7 ionospheric phase screen (IPS) on the differential 8 interferogram and in directional fluctuations in the relative 9 position of azimuth pixels on offsets maps. In this article, we 10 propose a procedure for estimating and removing 11 ionospheric contributions to surface displacement 12 measurements derived from L-band SAR data. We test the procedure on SAR data from the 28 May 2008 Sichuan Earthquake.

The applied corrections allow both a clearer interpretation of the surface rupture and a more accurate measurement of the surface displacement, which has important implications in earthquake modelling based on L-band SAR data.

Index Terms-radar, interferometry, ionosphere, earthquake

\section{INTRODUCTION}

25: thin the InSAR technique both the phase and the amplitude of the backscattered radar signals can be used 25 for measuring earth surface displacements and deformations. While DinSAR is based on the signal phase difference between two radar acquisitions ([1],[2]) and provides surface displacement values in the Line-of-Sight direction of the satellite (LOS), the sub-pixel correlation technique measures the sub-pixel offsets between two radar amplitude images both in the azimuth and LOS directions of the satellite (e.g. [3],[4]). The former technique is as accurate as a fraction of the employed radar wavelength and is sensitive to $\mathrm{mm}$ to $\mathrm{dm}$ surface displacement. The latter

technique is generally sensitive to ground displacements larger than 0.1 pixels, which is about $50 \mathrm{~cm}$ in the azimuth direction for a space-borne radar sensor such as the Phase Array L-band Synthetic Aperture Radar (PALSAR). These two techniques are complementary, particularly when LOS deformation gradients larger than one quarter of the wavelength per pixel cause interferometric signals to de-correlate. This might occur close to

Manuscript received April 20, 2009. This work was supported by the Research Division of BRGM. The data used in this study were provided by the Centre National d'Etudes Spatiales (CNES) through the Cellule d'Intervention et d'Expertise Scientifique et Technique (CIEST) agreement and the International Charter on Space and Major Disasters.

D. Raucoules and M. de Michele are with the BRGM (French Geological Survey), RNSC/RSC, 3 avenue C. Guillemin 45060 Orléans cedex 2, France (corresponding author phone: 33-2 386430 86; fax: 33- 2386433 37 ; e-mail: d.raucoules@ brgm.fr)

43 a seismic rupture, such as the $\sim 270 \mathrm{~km}$ long Sichuan earthquake 44 rupture where co-seismic slip reached up to 8 metres [5].

45 While L-band SAR signals are of particular interest in studying 46 earthquakes as it is less affected by temporal canopy changes 47 than C-band, it could be severely

48 affected by ionospheric heterogeneities occurring during the 49 synthetic aperture calculation (e.g. [6],[7]). Due to the 50 "dispersive" nature of the medium, the ionosphere refractive 51 index depends on the inverse of the square of the electromagnetic 52 frequency employed. Therefore, L-band SAR data are more 53 affected than C-band SAR.

54 The ionosphere influence on the SAR signal affects both azimuth 55 sub-pixel offsets and differential interferograms. The first bias 56 results from directional fluctuations in the relative sub-pixel 57 position of azimuth pixels, already reported in literature as 58 azimuthal "streaking" ([7]). The second bias results from relative 59 lengthening of the wave paths between two radar acquisitions 60 affecting the interferometric signal. As reported by recent studies 61 based on L-band InSAR on the Sichuan earthquake ([8],[9]), 62 ionospheric influences on the SAR signal appear to introduce 63 several difficulties for the retrieval of surface deformation from 64 both sub-pixel offset of radar amplitude images and differential 65 interferometry.

66 In this paper we focus on the ionospheric influences on the 67 SAR signal, assess their impact in the presence of co68 seismic surface displacement and try to propose a method 69 to estimate and remove their contributions both to sub70 pixel offset and to interferometric phase. We apply the 71 method to the 2008 Sichuan earthquake surface 72 displacement measurements.

\section{IONOSPHERIC EFFECTS ON SAR DATA}

\section{A. Interferometric phase}

The impact of the ionosphere on the interferogram is caused by the relative variation of the refractive index of the medium between the two radar acquisitions. The resulting propagation lengthening produces an interferometric phase shift.

This phase shift is related to the electron density variation, $n_{e}$ at height $h$ in eq.1. For a nadir-looking radar [11]:

$$
\Delta \Phi \approx-\frac{4 \pi}{c_{0}} \frac{40.28}{f} \Delta T E C
$$

where $\triangle T E C$ is the variation of the Total Electron Content (TEC), $c_{0}$ the speed of light, $f$ the signal frequency $(\mathrm{Hz})$. 
2 According to [7] atmospheric impact on C-band and L-band

3 InSAR results from radar signal phase modulation due to spatial

4 variation of the ionospheric propagation conditions during the 5 aperture time.

6 [11] derived the relation between ionospheric contribution to the

7 azimuth offset ( $\Delta x_{\text {iono }}$ ) and the ionospheric contribution to the

8 interferometric phase, which can be expressed as:
9

10

11

12

13

14

15

16

$$
\Delta x_{\text {iono }}=\alpha \frac{\partial}{\partial x}\left(\Delta \Phi_{\text {iono }}\right)
$$

Based on this relation we can estimate the interferometric phase correction, or IPS, starting from the azimuth offsets values [7]. We can then remove the IPS from the interferogram to enhance the coseismic deformation measure.

(1)

\section{AZIMUTH CORRECTION}

We observe that the azimuth streaks on the sub-pixel offset map show up with a preferential direction (figure 2a). Azimuth offsets are estimated on co-registred images (co-registration based on the adjustment of a bilinear model) of the interferometric pairs and therefore with identical geometry. In such conditions possible residual topographic effects are very limited (which is not the case with slant range offsets for with stereoscopic effect is not negligible even with perpendicular baselines of some tens of metres).

Over the Sichuan earthquake area the azimuth streaks direction is constant over a large spatial and temporal scale, at least during the concerned period (e.g. [8],[9]). The direction of the streaks seems to be constant for a given geographical area across different radar tracks. However, [7] who worked on polar areas noticed, in certain cases, along-track variation of this direction. The influence of the position respect to the magnetic poles has to be investigated for other test sites.

On the Sichuan area, the streaks strike $\sim$ N115E, while the earthquake ruptures strike $\sim \mathrm{N} 40 \mathrm{E}$ [5]. According to [14], we can observe that South China is located in an area affected by a strip of high electron density (related to the location of the geomagnetic equator) which main orientation roughly corresponds to $\sim \mathrm{N} 115 \mathrm{E}$. That could explain the direction of the streaks and the high values of ionospheric effects on the area.

Based on this peculiarity we improve the methodology firstly proposed by [7] by taking into account the spatial evolution of the azimuth streaks amplitude along their length over the entire radar image width.

In order to reduce the azimuth streaks, [8] proposed to cut the azimuth offset map into three sub-images within which the azimuth offset correction is approximated by a constant value along the streaks direction (i.e.the correction is constant by segments corresponding to the subimages) . This method provides a satisfactory correction to highlight the surface trace of the earthquake rupture and does not affect coseismic offset values in the near field. However, this approximation yields residual discontinuities at the sub-images boundaries.

Among the 1D low pass filters that could be used for this purpose, we propose to use single polynomial fits. In this paper, we approximate the azimuth streaks amplitudes by a third degree polynomial along the streak direction. After rotation of the image in order to align horizontally the streaks, each line is replaced by its third degree fit.This approximation fits the trend well enough
62 to remove most of the azimuth streaks without affecting high-

63 frequency small-scale offsets, such as near field offsets due to the 64 earthquake rupture. We test the methodology on two different 65 ALOS PALSAR tracks (table 1) acquired over the Sichuan area. 66 We first test the methodology on a radar track less affected by co67 seismic deformation (figure 2a) then we apply it to enhance the 68 coseismic rupture on a different track (figure $3 a / b$ ). We assume 69 that the computation of the $\alpha$ coefficient on the track less affected 70 by deformation is more reliable as the offset and phases are 71 mainly related to ionosphere and not deformation. Note that $\alpha$ 72 only depends on sensor parameters, so $\alpha$ is the same for both 73 frames.

74 In both cases, we compute sub-pixel offsets maps on full 75 resolution amplitude images by using the GAMMA routines 76 (http://www.gamma-rs.ch/), from which we subtract a linear 77 offset ramp (figure 2a). The linear offsets ramp is due to image 78 co-registration procedure and residual uncompensated orbits. The 79 ramp does not mask deformation but can be considered as a bias. 80 On the other hand, the objective of removing a ramp from offsets 81 is to obtain a result comparable to InSAR. In fact, InSAR is also 82 biased by a phase ramp on scales larger than the image coverage.

83 Such effects systematically affect InSAR results [13].

84 Figure 3a) and 3b) show an example of correction applied to the 85 azimuth offset map on track 473, concerned by the seismic slip 86 with values of up to $5 \mathrm{~m}$. We can notice that the deformation was 87 initially masked by the ionospheric contributions to azimuth 88 offset (figure 3a). After correction, the coseismic rupture is 89 enhanced and it can be mapped. Also we can retrieve the azimuth 90 component of the near field coseismic offset (about 1 pixel in the 91 azimuth direction, i.e. 3.6 metres), which is crucial for inverse 92 modelling of the earthquake

\section{COMPUTATION OF THE PHASE DERIVATIVE}

As pointed out in section 1, the contribution of the ionosphere to the azimuth offset can be associated to the along-track derivative of the interferometric phase. We will use this information to calculate the IPS and remove it from the coseismic interferogram.

As a prior processing step to estimate the phase correction, we compute the phase derivative on an extended area of track 471 where we infer no major surface deformation has occurred. In order to reduce noise, we applied a 20 pixel mean filter in the columns direction. Then, we compute the derivative by using eq.3. With this formulation, the derivative respect to the line index $i$ for a given pixel $(i, j)$ can be estimated on the complex interferogram without unwrapping.

$$
\frac{\partial}{\partial i}\left(\Phi_{i, j}\right)=W\left(W\left(\Phi_{i+1, j}\right)-W\left(\Phi_{i, j}\right)\right) \quad \text { with } \quad W(x)=x \quad[2 \pi] \quad \text { and }
$$

$W(x) \in[-\pi \mathrm{rad},+\pi \mathrm{rad}] \quad$ (3)

The benefits of the phase derivative image are twofold. Firstly, it allows us to confirm the validity of the azimuth offset correction estimation. In fact, similarities between the pattern of azimuth offset correction estimation (figure 2b) and the pattern of the phase derivative (figure 2d) confirms the validity of the former, as stated in eq. 2. On the other hand, the comparison of both results, allows us to estimate the proportionality coefficient $\alpha$ (eq. 2) necessary for IPS estimation. We examine both the standard deviations (table 1) and the scatter plot on a selected area of track 471 (figure 2d).

Considering the linear relation between the two datasets, the $\alpha$ value can be estimated as: 
1 $\alpha=\frac{\operatorname{std}\left(\Delta x_{i}\right)}{\operatorname{std}\left(\frac{\partial}{\partial i} \phi\right)}=30.8$ pixels/radian

3

$4 \beta=1 / \alpha=0.032 \mathrm{rad} /$ pixel

5

6 These parameters depend on geometric characteristics of a given

7 sensor in a given mode [11]. Therefore, once $\alpha$ and $\beta$ are

8 estimated on a given radar track/frame, one can use them for

9 correcting other radar frames acquired by the same sensor. This

10 point is of particular importance as we should not compare the

11 interferometric phase derivative with the azimuth offset

12 correction over an area that is affected by a high surface

13 deformation gradient since, in this case, the phase derivative

14 would be affected by surface deformation and the estimation of

$15 \alpha$ would be biased.

28 Where the $I$ are the results of the integration, $x$ is the ionospheric nterferogram on track 473. The IPS is the results of along-orbit integration of the ionospheric contribution to the azimuth offsets (obtained in section III), converted into the phase screen. The conversion from azimuth offsets to the phase screen is obtained by dividing offset values by coefficient $\alpha$.

$$
\begin{aligned}
I_{i, j} & =\sum_{i} \Delta x_{i, j} \\
\Delta \Phi_{\text {iono } i, j} & =\frac{1}{\alpha} I_{i, j}
\end{aligned}
$$

contribution to azimuth offsets, $i$ and $j$ are line and column indices respectively.

Figure 4a shows the extracted ionospheric contribution to the interferometric phase for track 473. In this case study, we can notice that the total ionospheric contribution to the interferometric phase corresponds to $\sim 4.0$ radians, which makes $\sim 0.6$ interferometric fringes or $\sim 7.5 \mathrm{~cm}$ apparent surface displacement in the LOS direction for PALSAR. For track 471 (figure 2e and 2f) the IPS ondulation is up to $\sim 18$ radians (i.e $~ 3$ fringes) equivalent to $34 \mathrm{~cm}$ displacement in LOS. Such a phase contribution can severely affect physical interpretations of the earthquake surface deformation based on L-Band interferograms over the mid-to-large scale deformation field, more precisely for wavelengths equal or larger than about $25 \mathrm{~km}^{-1}$. A more detailed study should be carried out to investigate the consequences of the ionosphere on shorter wavelengths.

Figures $2 \mathrm{~b}-2 \mathrm{f}$ and $4 \mathrm{~b}-4 \mathrm{c}$ compares the differential interferograms before and after correction. We can notice that the total deformation pattern is different. For track 471 we can observe a clear improvement of the interferogram such as decreasing of the fringe number and regularisation of the fringe pattern. A large wavelength bias of about 1 fringe still affects the corrected interferogram. It is probably due to uncompensated orbital ramp or a residual tropospheric contribution. At this stage, a coseismic interferogram is difficult to carry out. A validation would require a dense ground measurement network covering the entire study area. At the moment we are writing this manuscript, the GPS coverage on the area is not enough dense to allow a consistent validation ([15])
60 In this case study, the highest co-seismic slip (up to 8 61 metres) is located within $\sim 15 \mathrm{~km}$ of the rupture. Thus we 62 assume that the third-order polynomial used to calculate the 63 ionospheric contribution to the azimuth offsets has a minor 64 influence on the near-field co-seismic displacement values 65 measured by offsets (i.e. close to the rupture).

66 On the other hand, far-field deformations are usually smaller and

67 of longer wavelengths. In this case, the ionospheric contribution

68 to the interferometric phase should not be neglected for a correct 69 interpretation of the surface deformation.

70 Moreover, we have to notice that in another case where 71 deformation field produces long wavelength offsets in the same quantitative validation on the improvement made on the direction as the azimuth streaks, our methodology might result in an underestimation of the surface displacement as deformation signals would be more difficult to separate from the ionospheric contribution. On the other hand, given the nature of the ionospheric influence on the SAR signal, i.e. it concerns the derivative of the interferometric phase, independent TEC measures (such as by GPS, for instance) might not be adequately dense to resolve the mid wavelength ionospheric derivative and thus they would not be helpful in modelling and removing the ionospheric contribution to the azimuth offsets.
82

83

84

\section{CONCLUSION}

In this paper, we proposed a procedure for extracting ionospheric contributions to the SAR signal and we apply it to improve earthquake measurements based on PALSAR L-band SAR data over the Sichuan earthquake area. We used both sub-pixel correlation of radar amplitude images and radar interferometry. Based on the directionality of the azimuth streaking we defined an adaptive directional filtering method and approximated the ionospheric contribution to the azimuth offset. We then used this estimate to assess and remove the ionospheric contribution to the interferometric phase. The two following observations resulted from the presented study. Firstly, although they were initially severely affected, the azimuth sub-pixel offsets can be used both to precisely map the earthquake rupture and to measure the coseismic displacement in the near field.

Secondly, the ionospheric contribution to the interferometric phase (i.e. the IPS) can reach up to $\sim 15$ radians, equivalent to $\sim 28 \mathrm{~cm}$ apparent LOS displacement for ALOS PALSAR. This has important implications when using L-band interferometry to model the earthquake cycle.

\section{ACKNOWLEDGMENT}

The authors wish to thank Marie-Pierre Dion (ENS, Paris) for discussions. and J. Douglas for helping to improve the document.

\section{REFERENCES}

[1] A. K. Gabriel, R. M. Goldstein, and H. A. Zebker, "Mapping small elevation changes over a large area: differential radar interferometry”, J. Geophys. Res., vol 7, n², pp. 183-191, 1989 [2] D. Massonnet, and K. Feigl, "Radar interferometry and its application to changes in the Earth's surface, Rev. Geophys. Vol. 36, n 4 , pp. 441-500, 1998.

[3] R. Michel, JP Avouac, and J. Taboury, "Measuring ground displacements from SAR amplitude images: Application to the Landers earthquake”, Geophys. Res. Lett., vol. 26, n7, pp. 875$878,1999$.

[4] J. Hu, Z. Li, X. Ding and J. Zhu, "Two-dimensional CoSeismic Surface Displacements Field of the Chi-Chi Earthquake 
1 Inferred from SAR Image Matching.” Sensors, vol. 8, $n^{\circ} 10$, pp.

2 6484-6495, 2008

3 [5] X. Xixei, X. Wen, G. Chen, Q. Tian, J. Chen ,H. He, Y. He, 4 S. Yu , J. Ye, R. Zhou, G. Yu, L. Chen, Z. Li, C. Li, Y. An, Y. 5 Klinger, J. Hubbard and J.H. Shaw, "Surface ruptures, 6 aftershocks and geometry of the May $12^{\text {th }}$ 2008, Mw 8, 7 Wenchuan earthquake, China”, Geology, Submitted publication.

8 [6] S. Quegan and J. Lamont, "Ionospheric and tropospheric

9 effects on synthetic aperture radar performance", Int. J. Remote

10 Sens., vol. 7, n 4, pp. 525-539, 1986

11 [7] K.E Mattar, and A.L. Gray., "Reducing ionospheric electron 12 density errors in satellite radar interferometry applications”, Can.

13 J. Remote Sens., vol 28, n4 , pp. 593-600., 2002.

14 [8] M. de Michele, D. Raucoules, C. Lasserre, E. Pathier , Y.

15 Klinger, J. Van Der Woerd, J. de Sigoyer, and X. Xu, "The Mw

16 7.9, 12 May 2008 Sichuan earthquake rupture measured by sub-

17 pixel correlation of ALOS PALSAR amplitude images”, Earth

18 Planets Space accepted for publication

19 [9] T. Kobayashi, Y. Takada, M. Furuya and M. Murakami,

20 2008. "Ground deformation associated with the 2008 Sichuan

21 Earthquake in China, estimated using a SAR offset-tracking

22 method", presented at AGU fall meeting, San Francisco, USA,

23 December 15-19, 2008.

24 [10] P., Tapponnier and P. Molnar, “Active faulting and tectonics

25 in China”, J. Geophys. Res., vol 82, n²0, pp. 2905-2930, 1977.

26 [11] F. Meyer, R. Bamler, N. Jakowski, and T. Fritz,. "Methods

27 for small scale ionospheric TEC Mapping from broadband L

28 band SAR data”, presented at IGARSS06, Denver, USA, July 31-

29 August 04, 2006.

30 [12] S. Leprince, F. Ayoub, Y., Klinger and JP Avouac, “Co-

31 registration of optically sensed images and correlation (cosi-

32 corr): an operational methodology for ground deformation

33 measurements”, presented at IGARSS 07, Barcelona, Spain, July,

34 2007, pp. 2700-2702.

35 [13] Recommendations of the Fringe 2007, Fringe 2007,

36 30/11/2009 - 4/121/2009, Frascati, Italy,

37 http://earth.esa.int/workshops/fringe09/Fringr07_Summari

38 es_Recommendations.pdf

39 [14] H. Luhr , M. Rother, S. Maus, M. Mai and Cooke D.,

40 "The diamagnetic effect of the equatorial Appleton

41 anomaly; Its characteristics and impact on geomagnetic

42 field modelling”, Geophys. Res. Lett., vol 30, n 17 , pp

43 1906-1910, 2003

44 [15] Y. Zhang, W. Gong, and J. Zhang, ,2008, “Measuring

45 co-seismic deformation of the Sichuan earthquake by

46 satellite differential INSAR", Proc. SPIE, Vol. 7285,

47 72854F 


\section{TABLE I \\ ALOS PALSAR ACQUISITIONS USED FOR THIS STUDY}

\begin{tabular}{cccc}
\hline \hline Track & Date & Mode & $\begin{array}{c}\text { Pixel size } \\
\text { Range/Azimuth } \\
(\mathrm{m})\end{array}$ \\
471 & $\begin{array}{c}\text { 29/02/2008 } \\
\text { 31/05/2008 }\end{array}$ & Ascending & $4.68 / 3.15$ \\
473 & $17 / 02 / 2008$ & Ascending & $4.68 / 3.15$ \\
& $19 / 05 / 2008$ & & \\
\hline \hline
\end{tabular}

TABLE II

STANDARD DEVIATION OF THE PHASE DERIVATIVE AND THE AZIMUTHAL CORRECTION ON THE SOUTHERN AREA OF TRACK 473

\begin{tabular}{llc}
\hline \hline & standard deviation & mean \\
& & \\
InSAR Phase derivative & 0.013 rad & 0.0024 rad \\
$\begin{array}{llc}\text { Azimuth } \\
\text { correction }\end{array}$ & 0.37 pixels & 0.06 pixels \\
\hline \hline
\end{tabular}

Figure 1: Area of interest. Locations of PALSAR acquisitions used are identified by the dotted rectangles. WF= Wenchuan Fault; $B F=$ Beichuan Fault; PF= Guanxian-Pengguan Fault (modified from [10]).

Figure 2 a) azimuth sub-pixel offset map (track 471). Azimuth streaks are clearly visible. b) Ionospheric contribution to the azimuth offsets after directional polynomial fitting and linear trend removal c) Interferogram (track 471). Patterns with several fringes orientated in the 'streaks' direction are visible d) Along-orbit phase derivative (track 471). We observe the similarity with the azimuth streaks. The scatterogram between b) and d) data is plotted. It is consistent with a $\beta$ coefficient of $0.032 \mathrm{rad} /$ pixel (white line). e) IPS computed for track 471 . f) corrected interferogram. The dashed area corresponds to a noisy area on the azimuth offset image and therefore irrelevant correction.

Figure 3 a) azimuth offset map for track 473. b) Azimuth offsets map corrected for the ionosphere. The white line is the Sichuan earthquake surface rupture measure on the field (modified from [5]).

Figure 4 a) Ionospheric phase screen for track 473. b) Differential interferogram for Track 473 c) Corrected differential interferogram for track 473. 


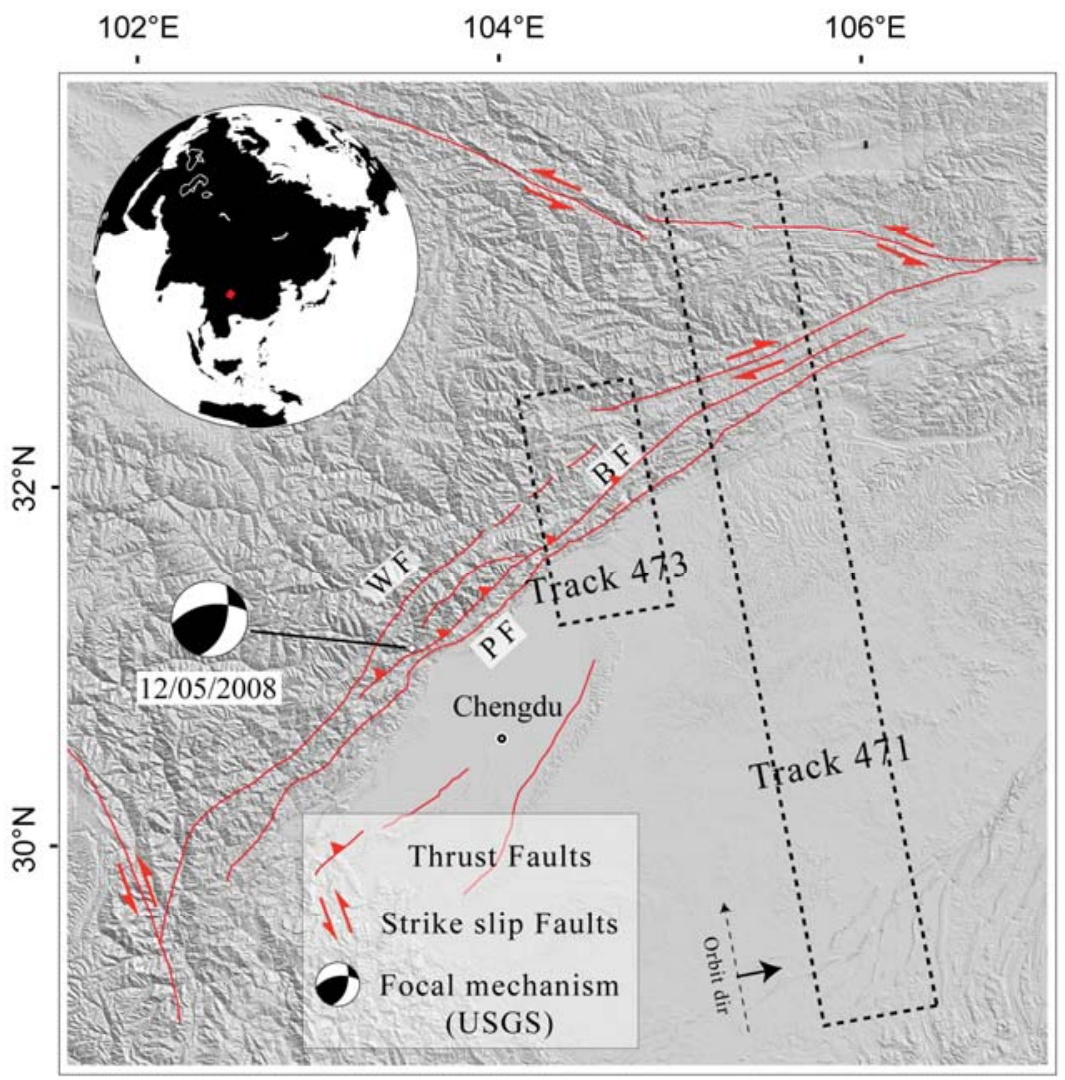



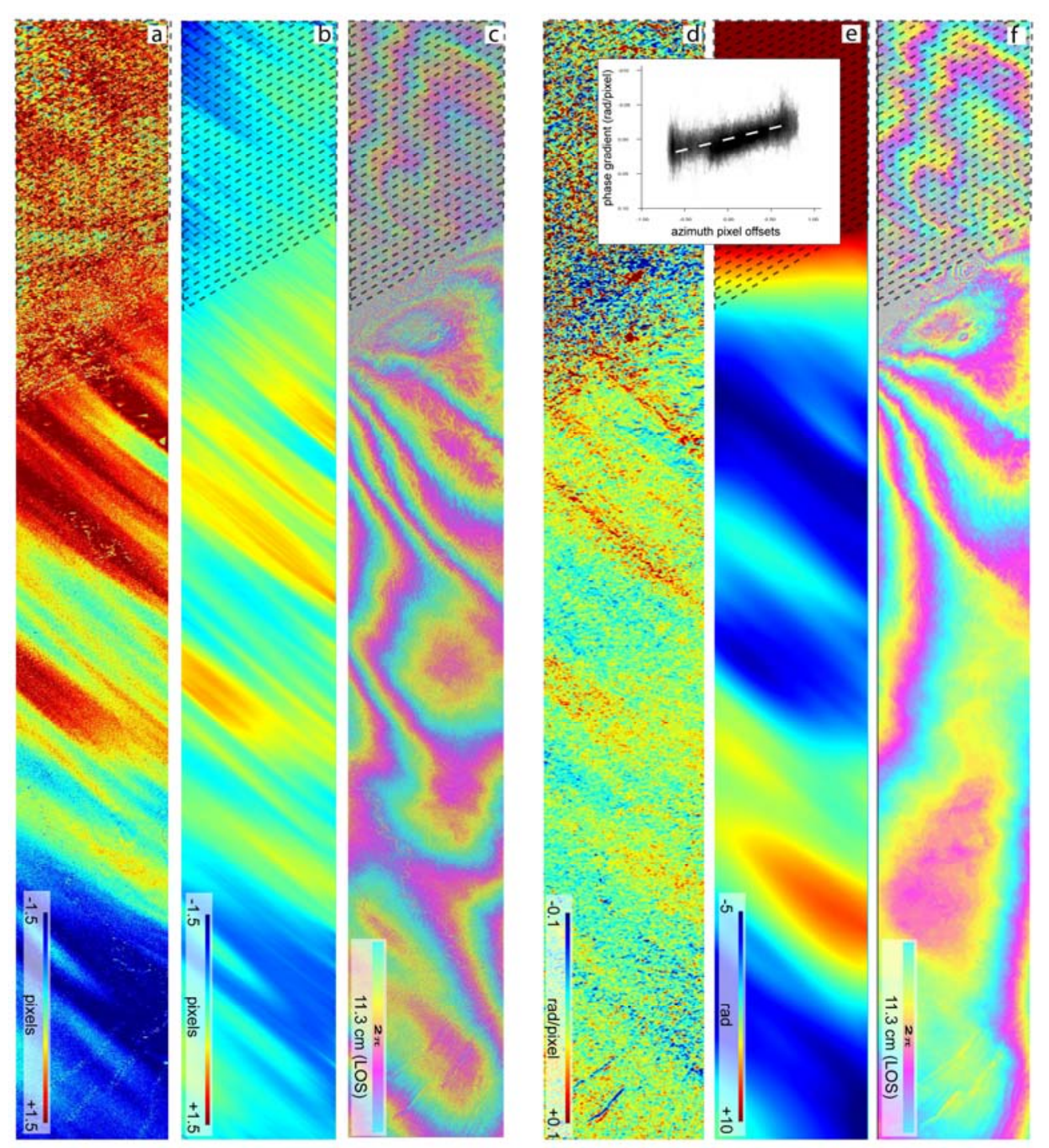


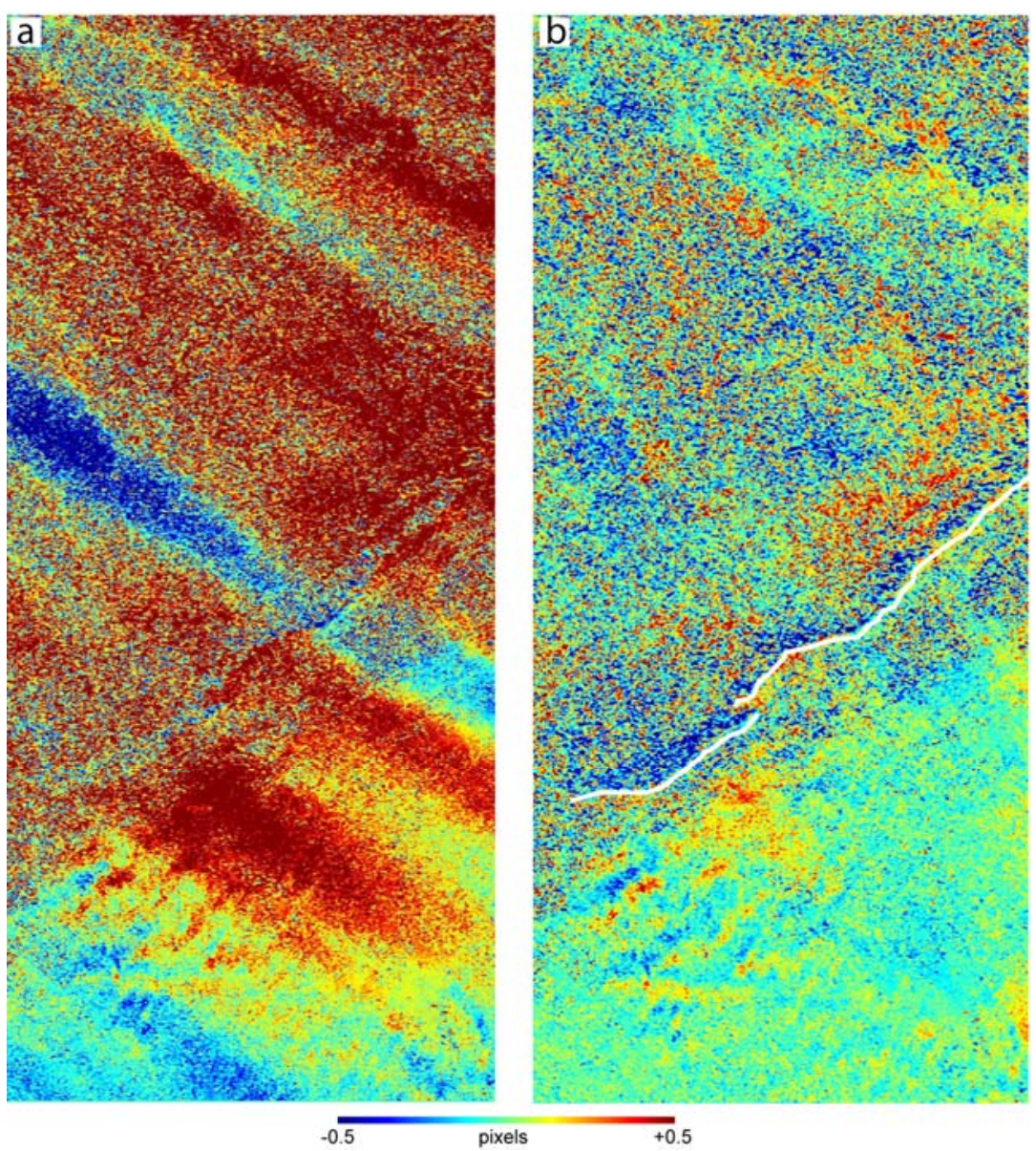




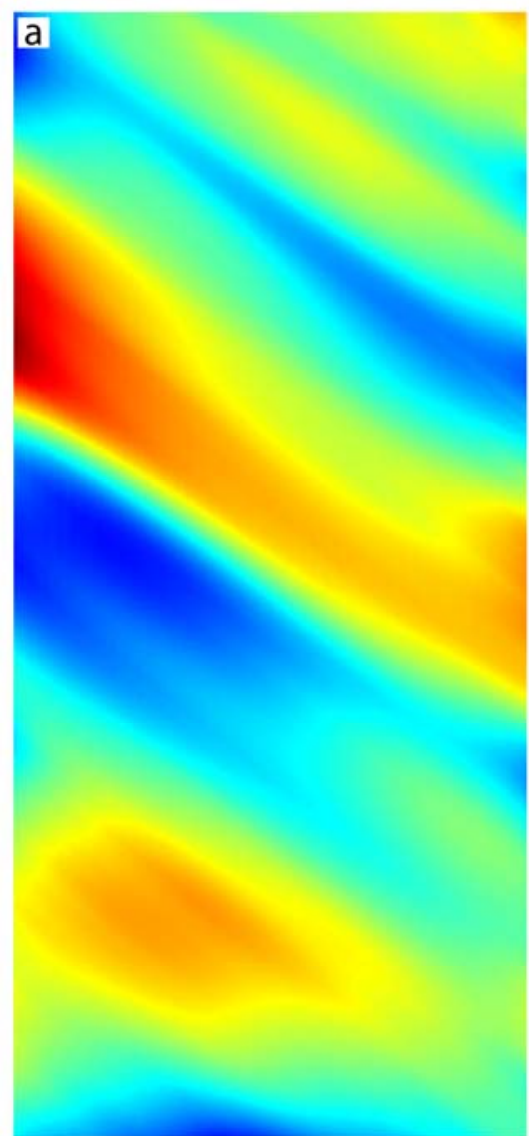

$-2.4$

$\mathrm{rad}$

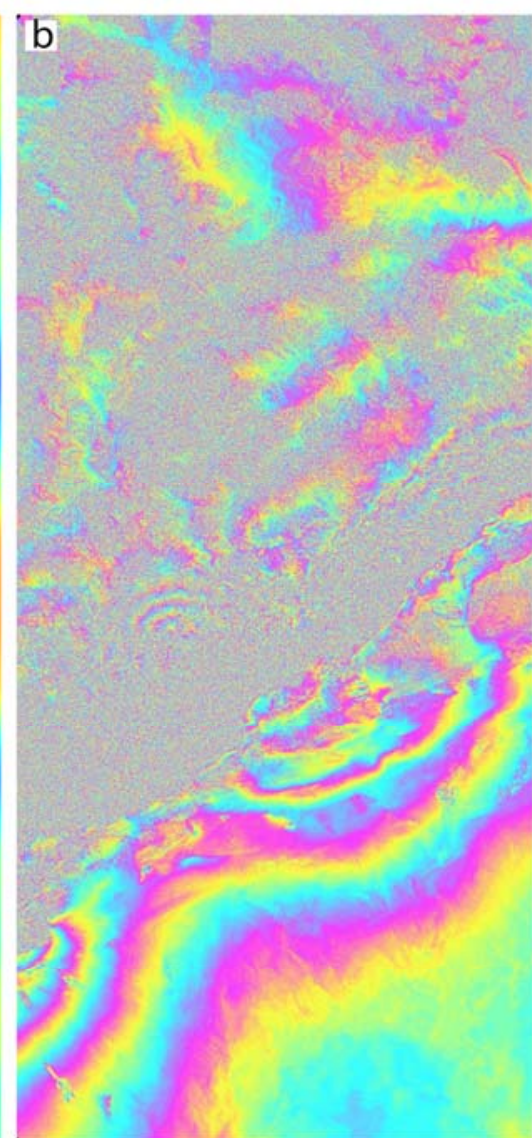

c

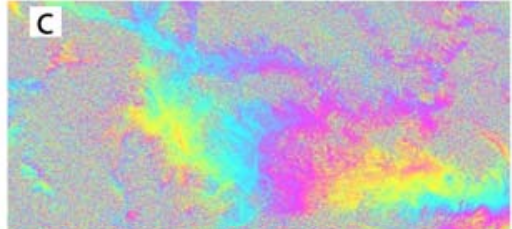

$113 \mathrm{~cm}$ (LOS) 